\title{
A Comparative Study of the Efficacy of an Improved Double-Lumen Tube Ventilation Control Device Versus A Conventional Device in Thoracic Surgery: A Randomized Controlled Trial
}

\section{Chang Liu}

Department of Anesthesiology, The Second Hospital of Jilin University

\section{Xin Wang}

Department of Anesthesiology, The Second Hospital of Jilin University

\section{Yue Wang}

Department of Anesthesiology, The Second Hospital of Jilin University

\section{Yuanyu Zhao}

Department of Anesthesiology, The Second Hospital of Jilin University Huiwen Guan

Department of Anesthesiology, The Second Hospital of Jilin University Xufang Sun ( $D$ 1013908844@qq.com )

Department of Anesthesiology, The Second Hospital of Jilin University

\section{Research Article}

Keywords: double lumen endotracheal tube connector, single-lung ventilation and, double-lung ventilation, double-lumen endotracheal tube

Posted Date: January 14th, 2021

DOI: https://doi.org/10.21203/rs.3.rs-137624/v1

License: (c) (i) This work is licensed under a Creative Commons Attribution 4.0 International License. Read Full License 


\title{
A comparative study of the efficacy of an improved Double-lumen tube ventilation control device versus a conventional device in thoracic surgery: a randomized controlled trial
}

\author{
Chang Liu, Xin Wang, Yue Wang, Yuanyu Zhao, Huiwen Guan and Xufang Sun*
}

\begin{abstract}
Background: To improve the conversion efficiency between single-lung and double-lung ventilation after Double-lumen tube(DLT) intubation, we invented a Y-shaped rotatable adjustment between three-lumen tube and double-lumen tube connector (YRC). The performance of YRC was studied with the traditional $Y$-shaped reconnector (YC) as the control.

Methods: A total of 60 adult patients who underwent thoracic surgery with double-lumen endotracheal tube insertion were included in the study. The subjects after endotracheal tube insertion were randomly divided into two groups: YRC connecting group (YRC group, $\mathrm{n}=30$ ) and a traditional connector connecting group (YC group, $n=30$ ), respectively. Primary endpoints were intubation time and conversion time of single-lung ventilation to double-lung ventilation. The secondary endpoints of this study were the internal gas volume of the two joints, airway pressure, and the time required for sputum aspiration during the operation.
\end{abstract}

Results: Intubation time in YRC group was shorter than that in YC group $(89.75 \pm 14.28 \mathrm{~s}$ vs $107.80 \pm 14.96 \mathrm{~s}, \mathrm{p}=0.00$ ). Compared with the $Y C$ group, the time required for ventilation conversion between single-lung and double-lung ventilation during the operation in the YRC group were significantly shortened $(3.60 \pm 1.20$ svs $9.05 \pm 2.53 \mathrm{~s}, \mathrm{p}=0.00)$. The internal gas volume in YRC $(17.20 \mathrm{ml})$ is smaller than YC $(24.12 \mathrm{ml})$. There was no statistical difference in airway pressure and the time required for sputum aspiration between YRC group and YC group (P> $0.05)$.

Conclusion: Compared with the traditional connectors, the YRC can improve the conversion efficiency between single-lung ventilation and double-lung ventilation with double-lumen endotracheal tube, save positioning time, and can be safely used for the anesthesia management of double-lumen endotracheal tube.

Trial registration: This clinical research was registered at the Chinese Clinical Trial Registry(www.chictr.org.cn,ChiCTR20000401 88), Date of registration: November 24, 2020.

Keywords: double lumen endotracheal tube connector; single-lung ventilation and; double-lung ventilation; double-lumen endotracheal tube 


\section{Background}

Double-lumen tube(DLT) is one of the most commonly used devices for realizing lung isolation and single-lung ventilation in thoracic surgery.[1].After endotracheal intubation, anesthesiologists are often required to control the breathing of patients to switch between double-lung ventilation and single-lung ventilation by adjusting the joint between endotracheal tube and breathing circuit. for example, when the endotracheal tube is positioned by auscultation and fibrobronchoscope, when pathological changes on the lung surface need to be better exposed during the surgery and when checking for air leakage at the stump after segmentectomy [2, 3, 4]. The double-lung ventilation connector (traditional connector, YC) provided by the existing dual lumen endotracheal tube (Shiley125-35, Covidien, the USA) which connects DLT with respiratory circuit should be used alternately with threaded pipe connector during ventilation mode conversion, resulting in low efficiency. In addition, ventilations need to be completely interrupted during the conversion process, and inhaled anesthetics are prone to leakage.

In order to improve the deficiencies of the traditional connector in the ventilation conversion of thoracic surgery, a $\mathrm{Y}$-shaped rotatable adjustment between three-lumen tube and double-lumen tube connector (YRC) was invented in the present study (which has been granted the patent for utility model with authorized notice No. CN 208481834 U). For this connector, the anesthesiologist can switch between the two ventilation modes by simply twisting the $Y$-valve on the surface. To compare the clinical performance of this connector with that of the traditional connector, we designed a randomized controlled trial. We hypothesize that the YRC may shorten the intubation time of endotracheal tube and improve the conversion efficiency of single-lung and double-lung ventilation modes. This will facilitate ventilation management during thoracic surgery.

\section{Methods}

This study was registered at www.chictr.org.cn (identifier:ChiCTR2000040188) on November 24, 2020. The study was approved by the Ethics Committee of the Second Hospital of Jilin University on November 23, 2020 (approval number of the Ethics Committee: 2020-138). Written informed consent was obtained from all patients. The study was performed in accordance with all relevant principles of the Declaration of Helsinki.

A total of 60 adult patients (ASA Grades I-II) who underwent thoracic surgery with doublelumen endotracheal tube insertion were included in the study. Exclusion criteria in this study included patients under the age of 18 years old, patients undergoing emergency thoracic surgery, patients who were pregnant, and patients who had contraindications for intubation of double-lumen endotracheal tube, such as laryngeal edema and acute laryngitis. Patients whose airway assessment before intubation was predicted as difficult airway, such as known airway mass, Mallampati grades III-IV, and severely limited head and neck movement, were also excluded [5, 6]. According to the random numbers generated by the computer, the patients were randomly divided into the YRC group $(n=30)$ or the YC group $(n=30)$. They were then stored in an opaque and digitally marked envelope, and disclosed before induction of total anesthesia.

Primary and secondary endpoints

Five anesthesiologists with more than ten years of clinical work experience and rich anesthesia experience in thoracic surgery and familiar with the use of two connectors were used to complete the anesthesia induction, DLTs intubation, positioning, sputum suction and other operations in the two groups of patients. The required indicators for this study were recorded by the same staff member who did not understand the study. The main results included intubation time and conversion time of single-to-double lung ventilation. Intubation time was defined as the time from passage of 
the catheter through the glottis to satisfactory positioning of the DLTs. The ventilation

conversion time was defined as the time required for conversion of double-lung ventilation to either-side single-lung ventilation or the time required for conversion of eitherside single-lung ventilation to double-lung ventilation. The internal gas volume of two connectors also needs to be measured. After positioning of DLTs in patients of two groups, peak airway pressure, plateau pressure and average airway pressure were recorded during $5 \mathrm{~min}$ of two-lung ventilation, and then they were recorded after switching to one-lung ventilation for $5 \mathrm{~min}$. During single-lung ventilation, the time required for sputum aspiration from the non-ventilated lung was recorded, which was defined as the time from the moment when the sputum aspiration tube was connected to the aspirator to the moment when the sputum aspiration tube completely left the opening of the double-chamber bronchial tube after the sputum aspiration. We also recorded the time required for sputum aspiration to the ventilated lung during singlelung ventilation, which is defined as the time from the moment when the sputum aspiration tube is connected with the aspirator to the moment when the patient resumes normal onelung ventilation after the sputum aspiration.

\section{YRC Introduction}

As shown in Figure. 1, the surface of YRC is provided with a $\mathrm{Y}$-shaped rotatable valve, and $y$-shaped mutually communicate pipelines inside the YRC can be driven by rotating the $y-$ shaped rotatable valve, so that the gas flow direction is controlled. The pipes b,c of the yvalve are at an angle of 90 and their corresponding internal pipes are also at an

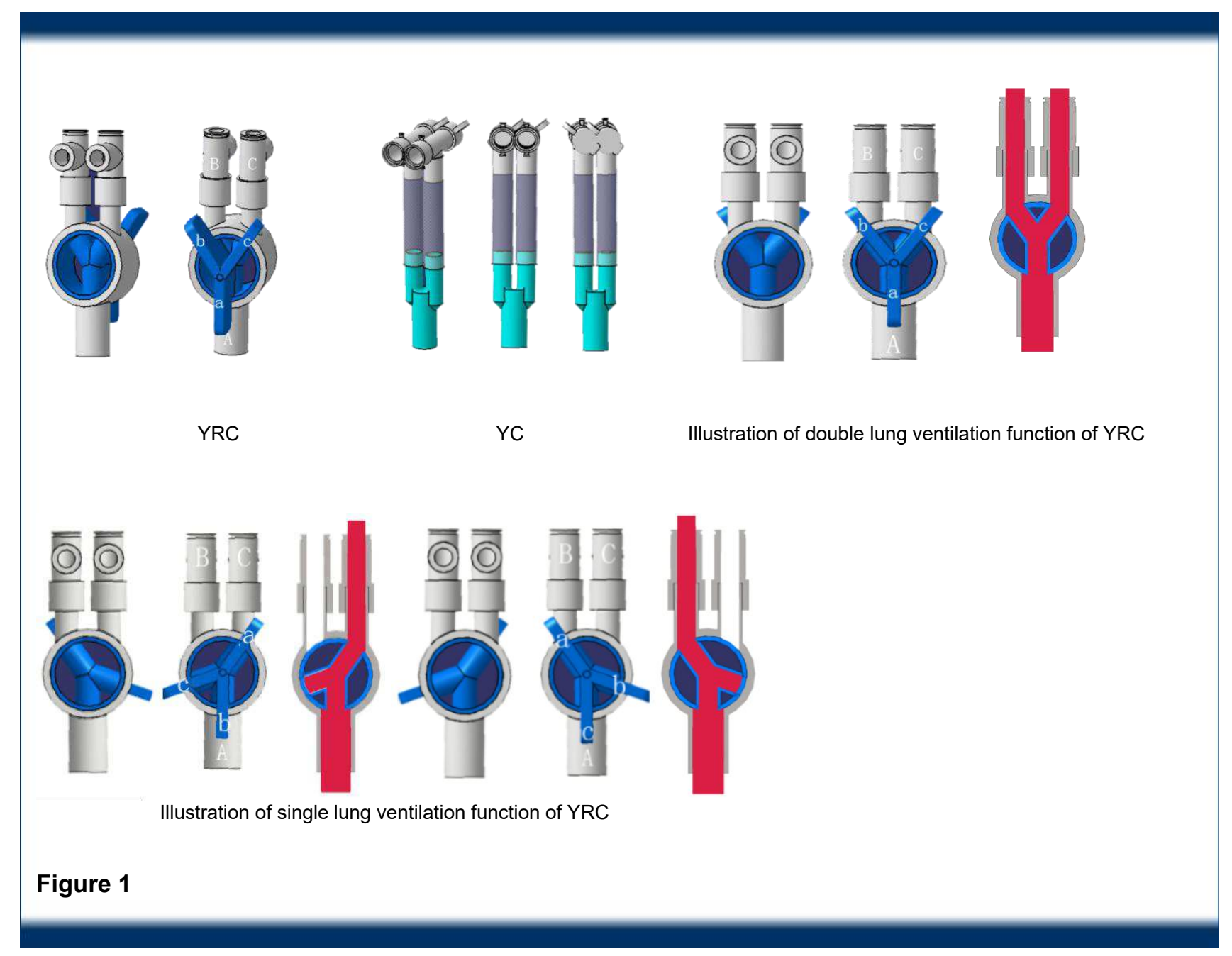


angle of 90 . The pipes $b$ and $c$ form an angle of 135 with a, and corresponding internal pipe of pipe a also form an angle of 135. However, YC can only realize double-lung ventilation after connecting the double-lumen bronchial tube with the respiratory circuit, but can not carry out single-lung ventilation (Figure. 1).

When two lungs are ventilated, the $b$ and $c$ ends of the $Y$-valve point to the $B$ and $C$ ends of the double-lumen endotracheal tube, respectively, and the a end points to the breathing circuit end A (Figure. 1). When singlelung ventilation was performed, the a end points of the $\mathrm{Y}$-valve pointed to the ventilated side of the double-lumen endotracheal tube. If it pointed to the $B$ end, $C$ pointed to the respiratory circuit end $A$, and the $b$ end was closed. At this time, the open side of the double-lumen endobronchial tube connected with the $B$ end was ventilated, while the open side of the double-lumen endobronchial tube connected with the $C$ end was not ventilated. If it points to end $C$, end $b$ points to end $A$ of breathing circuit, and end $\mathrm{C}$ is closed. At this time, the open side of the double-lumen endobronchial tube connected with the $C$ end was ventilated, while the open side of the double-lumen endobronchial tube connected with the B end was not ventilated. (Figure. 1). Therefore, when performing the conversion between the two ventilation modes, only the $\mathrm{Y}$ shaped valve needs to be rotated for realization. The clinical application effect of YRC and YC is shown in Figure 2.

\section{Research process}

Electrocardiograms, invasive arterial blood pressure, pulse oximetry, and bispectral index were routinely monitored after all patients entered the operating room. Lidocaine cream was applied to the cuff at the front of the catheter for lubrication and superficial anesthesia. Induction of general anesthesia was achieved by sequential intravenous administration of midazolam $(0.05-0.1 \mathrm{mg} / \mathrm{kg})$, propofol $(2-3 \mathrm{mg} / \mathrm{kg})$, sufentanil $(0.5 \mathrm{ug} / \mathrm{kg})$, and cis-atracurium $(0.15-0.2 \mathrm{mg} / \mathrm{kg})$. During induction, patients were given pure oxygen mask ventilation for 3 minutes, and then endotracheal intubation was performed. The anesthesiologist exposed the glottis using a visual laryngoscope, and the blue cuff at the bronchial end of the double-lumen tube completely passed through the glottis and then pulled out the tube core provided with the double-lumen tube. Then, according to the

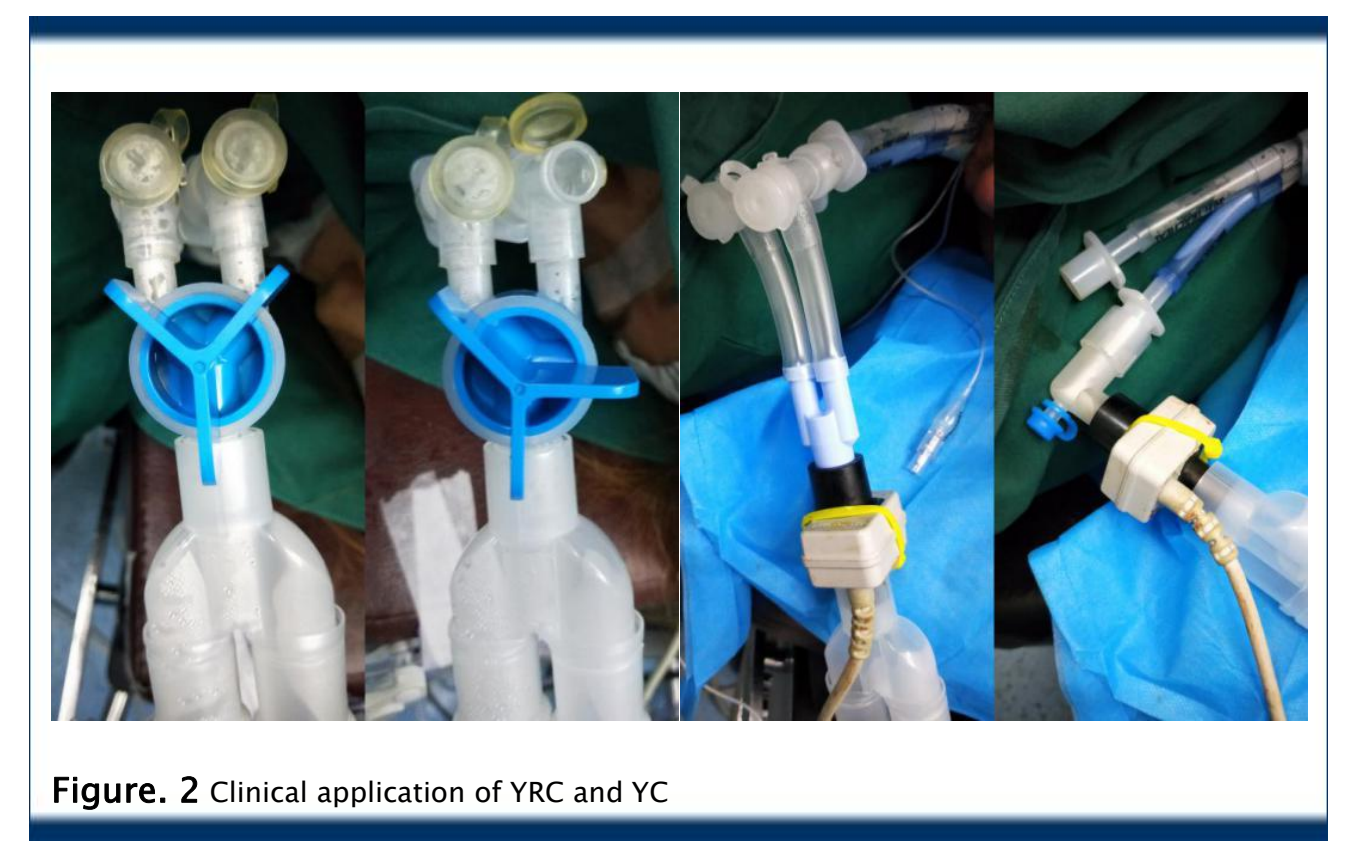


direction of intubation, the double-lumen tube is rotated to advance toward the bronchus until it feels moderate resistance, and then the two sets of balloons are inflated respectively. After connecting with YRC, YRC group underwent ventilation mode conversion by rotating $Y$-valve, while in $Y C$ group, the ventilation mode was changed by alternately using $Y C$ and threaded pipe connector. In two groups, the patient was first ventilated with two-lung ventilation or one-lung ventilation by manual breathing, and the respiratory sounds were auscultated. By preliminarily adjusting the position of the double-chamber tube, both lung respiratory sounds were clear during double-lung ventilation, while during one-lung ventilation, the respiratory sounds in the ventilated side were clear (especially in the upper lobe) and the respiratory sounds were completely inaudible on the non-ventilated side. Fiberoptic bronchoscopy was then applied to determine the position of the double lumen tube. Finally, two-lung mechanical ventilation was performed, and the double-lumen tube was fixed. Before pleural opening, one-lung ventilation was used. After the posture was changed, the catheter position was confirmed and adjusted by fibrobronchoscopy. After anesthesia induction, continuous intravenous target-controlled infusion of propofol (the set target concentration of $2-4 \mathrm{ug} / \mathrm{mL}$ ) and remifentanil (0.2-0.5 ug $/ \mathrm{kg} . \mathrm{min})$ maintained BIS within the range of $40-60$, and intermittent injection of cis-atracurium to maintain appropriate muscle relaxation. During two-lung ventilation, the tidal volume of the ventilator was adjusted to $7 \mathrm{ml} / \mathrm{kg}$, with the frequency of 12-14 times/min. During one-lung ventilation, the tidal volume was adjusted to $5 \mathrm{ml} / \mathrm{kg}$ with a frequency of $14-$ 16 times/min, and the PEEP was adjusted to 8 ,

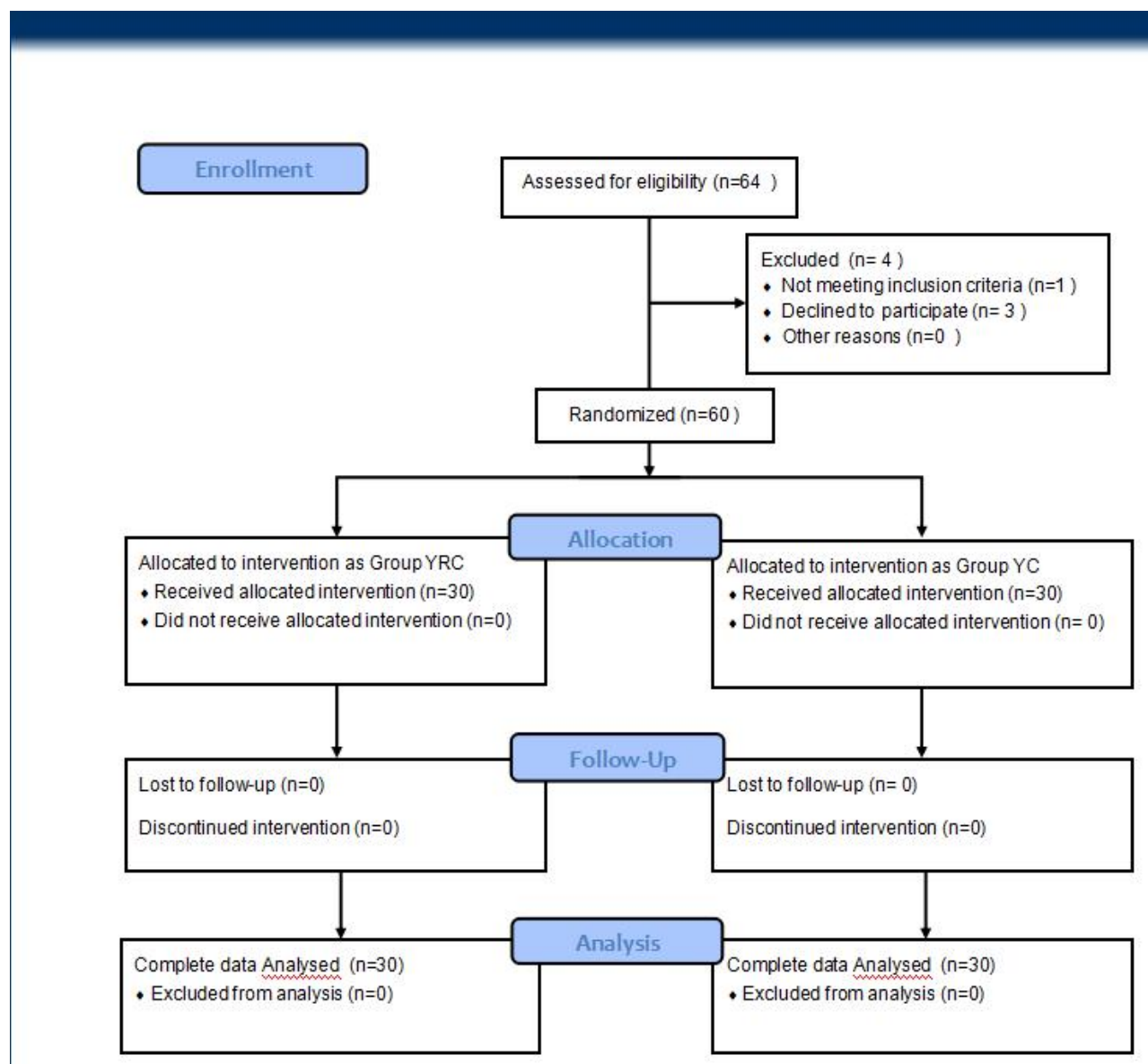

Figure 3. CONSORT of flow diagram 
maintaining the end-tidal partial pressure of carbon dioxide of $35-45 \mathrm{mmHg}$.

\section{Statistics analysis}

Statistical analysis was performed using SPSS25.0 software. The measurement data conforming to normal distribution were expressed as mean \pm standard deviation $(\bar{X} \pm S)$. Independent sample $t$ test was used for comparison between groups, and the enumeration data were subjected to $\mathrm{x}$ test. $\mathrm{P}<$ 0.05 indicated statistically significant.

\section{Results}

Preliminary qualification assessments were performed in 64 patients in this study. Three patients refused to sign the study consent form, and one patient was excluded due to the preoperative assessment of difficult airway. Finally, 60 patients participated in the study analysis (Figure 3 ), including 44 cases of lung mass, 2 cases of pneumothorax, 5 cases of mediastinal tumor, 3 cases of esophageal lesions, 3 cases of empyema, 1 case of pericardial effusion, 1 case of rib malignant tumor, and 1 case of rib fracture. There was no statistically significant difference in biometric data between the two groups (Table 1).

The results showed that YRC, compared with YC, could improve the conversion efficiency of single-lung ventilation and double-lung ventilation with dual lumen endotracheal tube, and reduce the intubation time, but there was no difference in the impact on the sputum suction operation between two groups. The intubation time in the YRC group was shorter than that in the YC group $(89.75 \pm 14.28 \mathrm{~s}$ VS $107.80 \pm 14.96 \mathrm{~s}, \quad p=0.00$ ) (Figure 4). The ventilation conversion time in the YRC group was shorter than that in the $Y C$ group $(3.60 \pm 1.20$ s VS $9.05 \pm 2.53 \mathrm{~s}, \mathrm{p}=0.00)$ (Figure 4). The sputum aspiration time in the YRC group

Table 1 Patient characteristics

\begin{tabular}{llll}
\hline Variable & YRC group $(\mathrm{n}=30)$ & YC group $(\mathrm{n}=30)$ & P value \\
\hline Gender(male/females) & $15 / 15$ & $14 / 16$ & 0.800 \\
Age(years) & $58.7 \pm 11.8$ & $56.5 \pm 10.5$ & 0.712 \\
Height $(\mathrm{cm})$ & $166.3 \pm 8.9$ & $165.9 \pm 8.2$ & 0.836 \\
Weight(kg) & $65.5 \pm 12.4$ & $63.9 \pm 11.2$ & 0.633 \\
ASA (I/II) & $1 / 29$ & $2 / 28$ & 0.561 \\
DLT design used(left/right) & $22 / 8$ & $20 / 10$ & 0.581 \\
\hline
\end{tabular}

Table 2

\begin{tabular}{llll}
\hline Variable & YRC & YC group $(\mathrm{n}=30)$ & P value \\
\hline Intubation time (S) & $89.75 \pm 14.28$ & $107.80 \pm 14.96$ & $0.00^{\mathrm{b}}$ \\
Conversion time(S) & $3.60 \pm 1.20$ & $9.05 \pm 2.53$ & $0.00^{\mathrm{b}}$ \\
$\begin{array}{l}\text { Sputum aspiration time to the } \\
\text { non-ventilated lung during one- } \\
\text { lung ventilation (S) }\end{array}$ & $8.79 \pm 1.95$ & $9.64 \pm 1.77$ & 0.081 \\
$\begin{array}{l}\text { Sputum aspiration time to the } \\
\text { non-ventilated lung during one- } \\
\text { lung ventilation (S) }\end{array}$ & $13.56 \pm 1.81$ & $14.11 \pm 1.68$ & \\
Gas volume comparison(ml) & 17.20 & & 0.304 \\
\hline
\end{tabular}


was not significantly different from that in the YC group $(8.79 \pm 1.95 \mathrm{~s}$ VS $9.64 \pm 1.77 \mathrm{~s}, \mathrm{P}=$ $0.081>0.05,13.56 \pm 1.81 \mathrm{~s}$ VS $14.11 \pm 1.68 \mathrm{~s}, \mathrm{P}$ $=0.304>0.05)$ (Table 2).

The gas volume inside the joint is measured three times. The measurement method is to seal the ends of the two connectors connected to the breathing circuit with adhesive tape, and inject water into them from the end connected to the endotracheal tube until the connectors are completely filled with water, and measure the milliliters of injected water, that is, the internal gas volume value of the two connectors. The results showed that the average volume of gas in YRC was $17.20 \mathrm{ml}$, and that in YC was $24.12 \mathrm{ml}$, which indicated that YRC could reduce the mechanical dead space in breathing circuit. There was no difference in peak airway pressure, platform pressure or average airway pressure between the two groups during one-lung ventilation and two-lung ventilation for $5 \mathrm{~min}$, indicating that YRC, like YC, could ensure the overall airway tightness and airway safety during mechanical ventilation $(P>0.05)$ (Table 3).

\section{Discussion}

In thoracic surgery, double-lumen tube (DLT) is required to separate the bilateral thoracic ventilation [7]. The traditional connector (YC) connecting the double-lumen endotracheal tube to the respiratory circuit needs to be used alternately with the single-lumen connector for the single-to-double lung ventilation conversion. The deficiency of $Y C$ is that the operation of ventilation conversion process is cumbersome, and the connectors may fall off or be lost in the process of repeatedly plugging and unplugging. If the connectors are not found in time, the sealability of the whole respiratory

Table 3

\begin{tabular}{llccccc}
\hline \multirow{3}{*}{ Group } & \multicolumn{3}{c}{ Double-lung ventilation } & \multicolumn{3}{c}{ Single-lung ventilation } \\
\cline { 2 - 7 } & $\begin{array}{c}\text { Ppeak } \\
\left(\mathrm{cmH}_{2} \mathrm{O}\right)\end{array}$ & \multicolumn{1}{c}{$\begin{array}{c}\text { Pplat } \\
\left(\mathrm{cmH}_{2} \mathrm{O}\right)\end{array}$} & $\begin{array}{c}\text { Pmean } \\
\left(\mathrm{cmH}_{2} \mathrm{O}\right)\end{array}$ & $\begin{array}{c}\text { Ppeak } \\
\left(\mathrm{cmH}_{2} \mathrm{O}\right)\end{array}$ & $\begin{array}{c}\text { Pplat } \\
\left(\mathrm{cmH}_{2} \mathrm{O}\right)\end{array}$ & $\begin{array}{c}\text { Pmean } \\
\left(\mathrm{cmH}_{2} \mathrm{O}\right)\end{array}$ \\
\hline YRC group(n=30) & $16.05 \pm 2.19$ & $14.10 \pm 1.87$ & $6.21 \pm 0.79$ & $24.68 \pm 3.29$ & $21.50 \pm 2.65$ & $9.90 \pm 1.22$ \\
YC group(n=30) & $15.95 \pm 2.34$ & $13.79 \pm 1.55$ & $5.92 \pm 0.71$ & $23.96 \pm 2.60$ & $20.98 \pm 2.02$ & $9.26 \pm 0.98$ \\
P value & 0.380 & 0.104 & 0.310 & 0.206 & 0.094 & 0.078 \\
\hline
\end{tabular}

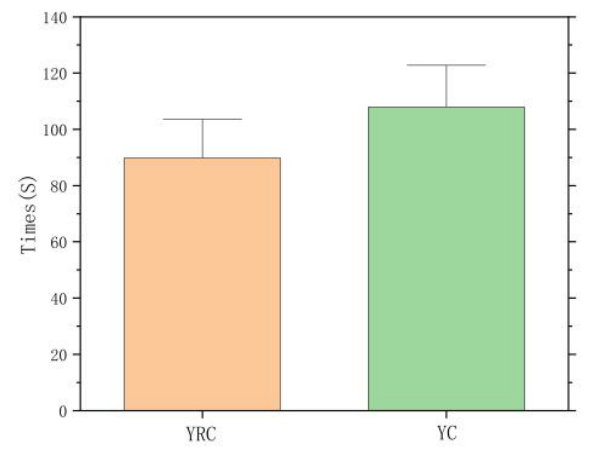

Intubation time chart

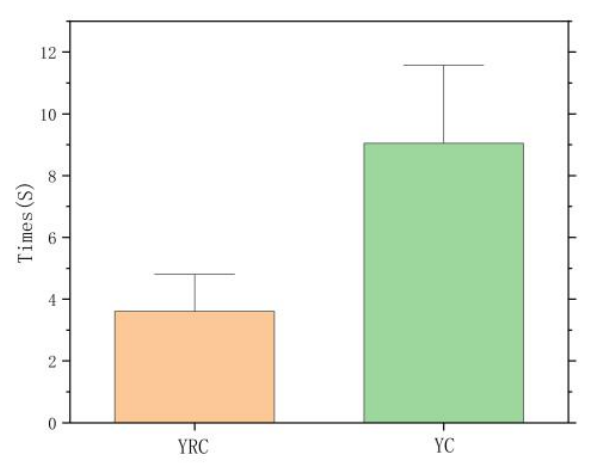

conversion time chart 
circuit during anesthesia will be affected, resulting in hypoxia for patients, and even a threat to life safety [8]. If the connector is lost, the smoothness of the ventilation conversion for the patient is reduced, and the operation progress is delayed. At the same time, repeatedly plugging and unplugging during inhalation anesthesia will cause a large amount of inhaled anesthetics to leak from the respiratory circuit, affect the stability of the anesthesia depth of patients, and pollute the air in operation rooms.

In order to solve the above-mentioned problems, the author of this paper invented YRC, which does not need to completely disconnect the respiratory circuit during single-to-double lung ventilation conversion, and the ventilation mode can be switched by only rotating the $Y$ type valve, with simple operation.

When anesthesiologists use auscultation to locate the endotracheal tube after tracheal intubation, they need to frequently switch the ventilation mode. From the results of this study, we can see that YRC, compared with YC, greatly saves the intubation time of double-lumen endotracheal tube and the ventilation switching time and reduces the no-ventilation time of patients. For patients with poor lung function and poor oxygen storage, such as COPD and pulmonary fibrosis, it is crucial to shorten the time without ventilation during the intubation [9, 10], which improves the safety of the intubation process. And the internal gas volume of YRC is smaller than that of $Y C$, which reduces the mechanical dead space in the breathing circuit, thus reducing the mechanical compliance of the whole machine, reducing the ventilation loss and improving the ventilation efficiency of the whole machine $[11,12]$.

Y-shaped rotatable valve core is the core knowledge value of the invention, which not only realizes the random conversion of single and double lung ventilation, but also has the advantages of saving time and labor in operation performance. It is the same as the traditional connector in airtightness and safety, with low cost and disposable use, which can ensure the health and safety of respiratory system.

In addition, although this study was randomised, there are still certain limitations that deserve our attention.First of all, all anesthesiologists participating in the study were not blinded to the connector used, but it is difficult to solve this problem in a comparative study evaluating the application of the two connectors in thoracic surgery.However, the recorded time and other data were completed by the same staff who did not understand the experiment, and the recorded indicators were objective.Another limitation is that due to the limitations of conditions, all patients participating in this study use the method of intravenous anesthesia, which cannot show the differences in the effects of these two connectors on the depth of anesthesia during inhalation anesthesia. This is worthwhile Further discussion.

\section{Conclusions}

In summary, the YRC has certain practicability, effectively improves the ventilation mode conversion efficiency, and is an innovative instrument worthy of promotion.

\section{Abbreviations}

DLT: Double Lumen Tube; BIS: Bispectral Index Monitoring;

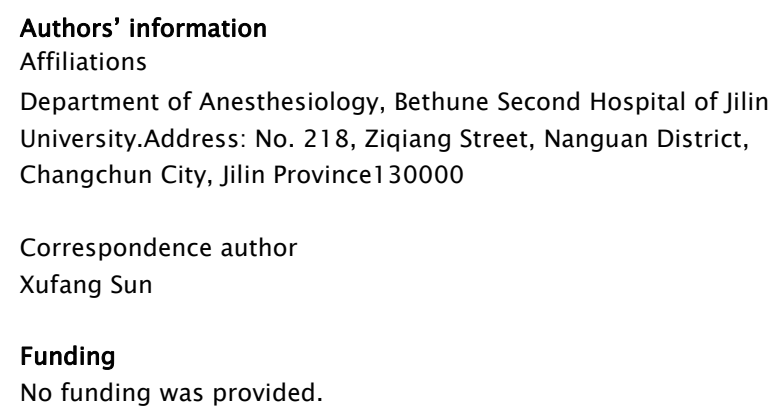
www.chictr.org.cn(identifier:ChiCTR2000040188). The study was approved by the Ethics Committee of the Second Hospital of Jilin University on November 23, 2020 (approval number of the Ethics Committee: 2020-138). Written informed consent was obtained from all patients.

Competing interests

The authors declare that they have no competing interests. 
Authors' contributions

LC,WY, andGHW were reponsible for the study design and planning.LC and ZYY analyzed the data and drafed the manuscript as well .SXF and WX,also joined the work of study design and revise the manuscript.All authors read and approved the dinal manuscript.

\section{Acknowledgements}

The authors would like to thank Mr. Fenghao Sun from Northeast Dianli University for his technical guidance.

\section{References}

1. Cohen E. Recommendation for airway control and difficult airway management in thoracic anesthesia and lung separation procedures. are we ready for the challenge? Minerva Anesthesiol.2009;75:3 - 5.

2. BERNASCONI F, PICCIONI F. One-lung ventilation for thoracic surgery: current perspectives. Tumori.2017, 103(6):495-503.

3. JUNG D M, AHN H J, JUNG S H, et al. Apneic oxygen insuflation decreases the incidence of hypoxemia during one-lung ventilation in open and thoracoscopic pulmonary lobectomy:A randomized controlled trial.Thorac Cardiovasc Surg.2017, 154(1):360-366.

4. Hannallah MS, Benumof JL, McCarthy PO, et al. Comparison of three techniques to inflate the bronchial cuff of let polyvinylchloride double-lumen tubes. Anesth Analg.1993,77(5):990-994.

5. Shiga T, Wajima Z, Inoue T, Sakamoto A. Predicting difficult intubation in apparently normal patients: a meta-analysis of bedside screening test performance. Anesthesiology. 2005;103:429-37.

6. Apfelbaum JL, Silverstein JH, Chung FF, Connis RT, Fillmore RB, Hunt SE, et al.Practice guidelines for postanesthetic care: an updated report by the American Society of Anesthesiologists Task Force on Postanesthetic care. Anesthesiology. 2013;118(2):291-307.

7. Effects of the anesthesiologist's experience on postoperative hoarseness after double-lumen endotracheal tube intubation: a single-center propensity score-matched analysis.2020, 20(1):278278.

8. 苏蓬.麻醉机呼吸回路密闭性的检测与维修.医疗装备,2018,31(14):141. 9. Schatier PR, Luna J. Ghiatas AA, et al. pulmonary fuction after laparoscopic cholecysteclclrny Surgery. 1993,1(14):389.

10. Ledowski T. Bein B. Hanss R, et al. Neurodenocrine stress response and heart rate variability: a comparison of total intravenous versus balanced anesthesia.Anesth Analg, 2005, 101:1700.

11. 黄卫平. 麻醉的风险评估与安全管理.中医药管理杂志, 2018,26(09): 166167.

12. 杨翌, 姚欣. 死腔通气的计算及其临床应用. 中华结核和呼吸杂 志,2016,39(09):727-729. 
Figures

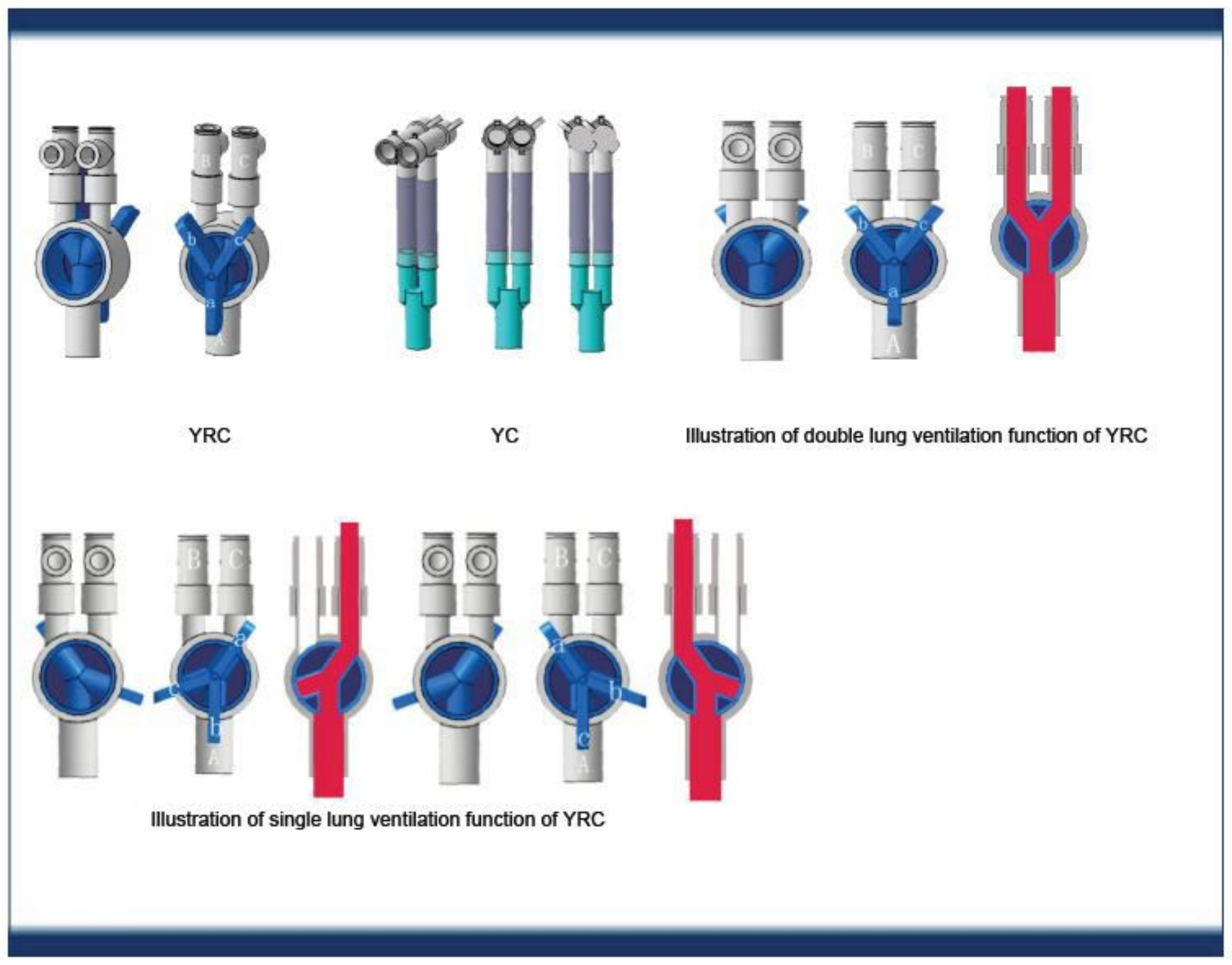

Figure 1

As shown in Figure. 1, the surface of YRC is provided with a Y-shaped rotatable valve, and y-shaped mutually communicate pipelines inside the YRC can be driven by rotating the $y$-shaped rotatable valve, so that the gas flow direction is controlled. 


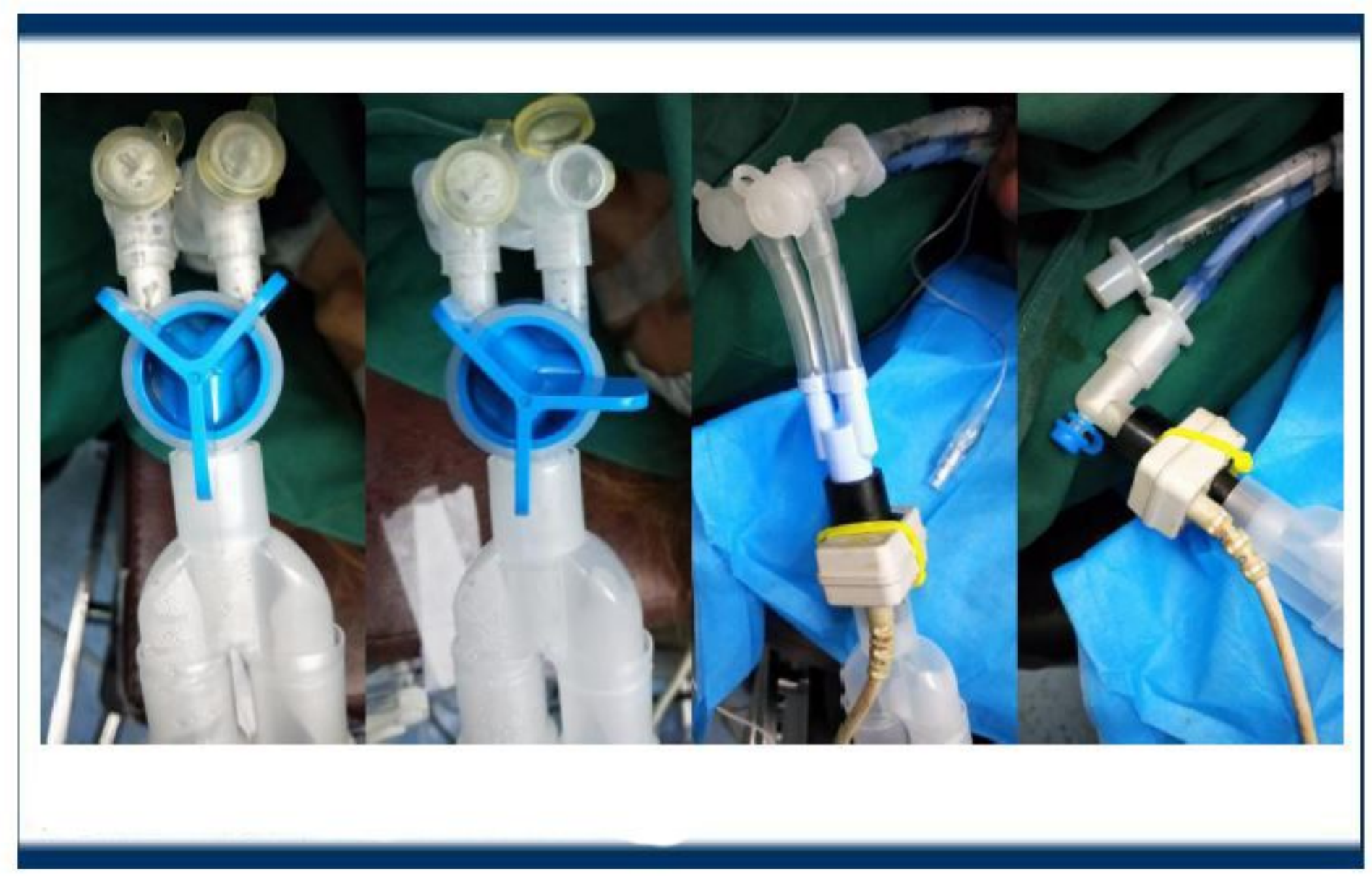

Figure 2

Clinical application of YRC and YC 


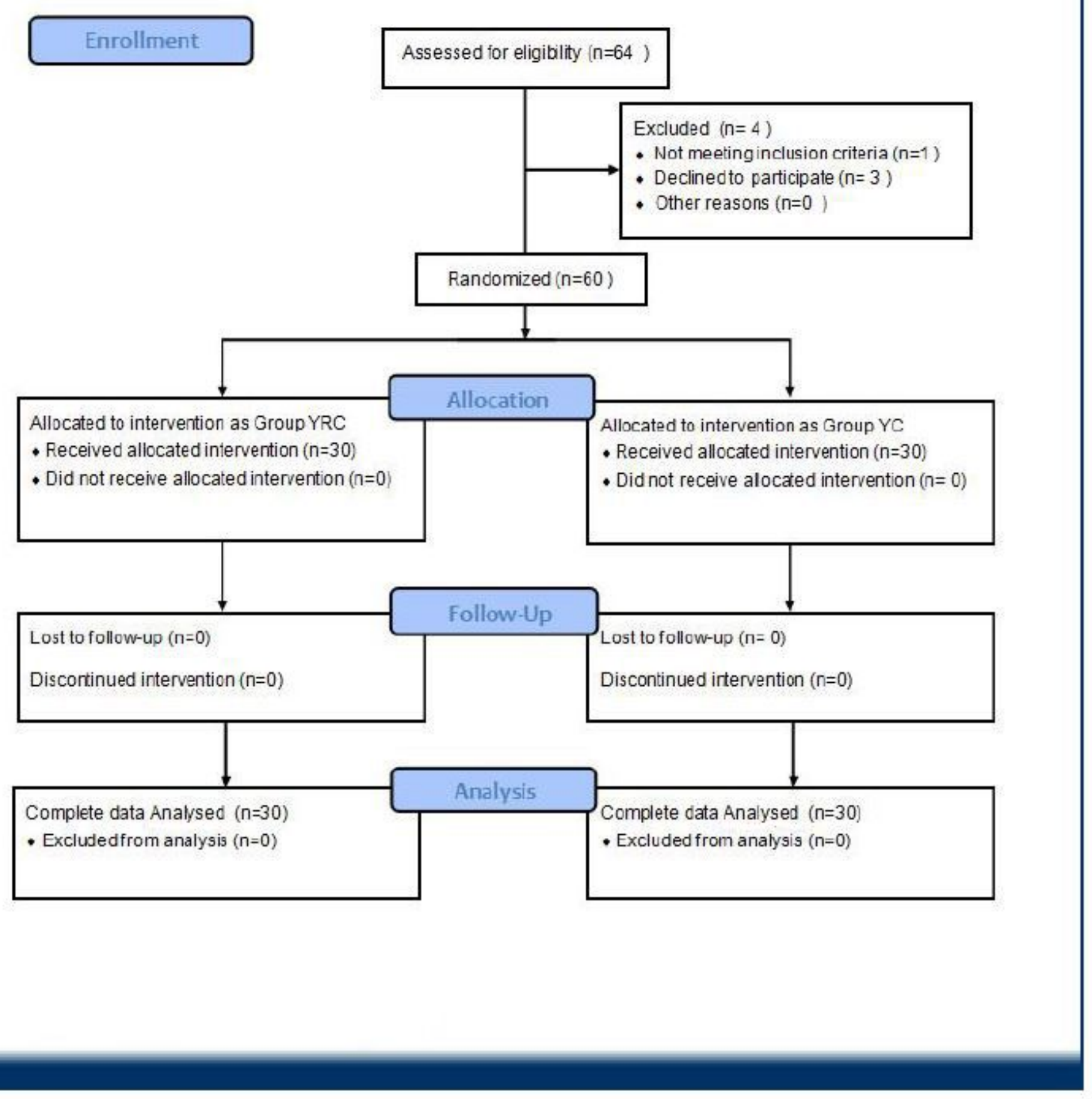

Figure 3

CONSORT of flow diagram 


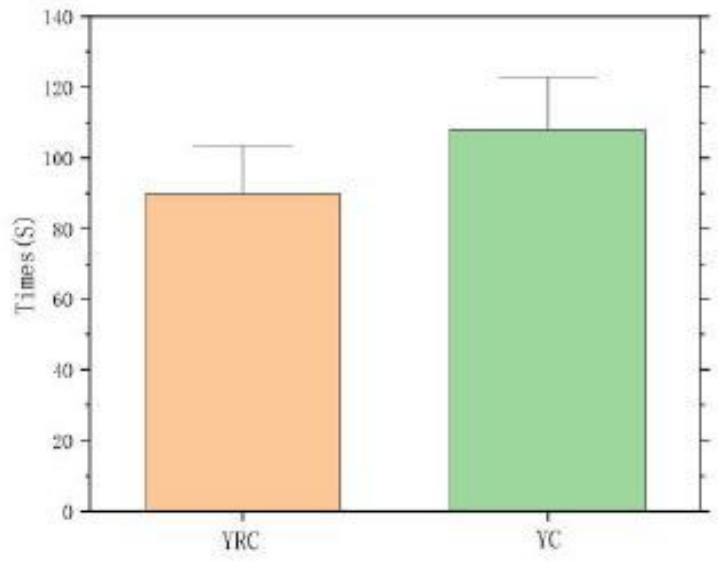

Intubation time chart

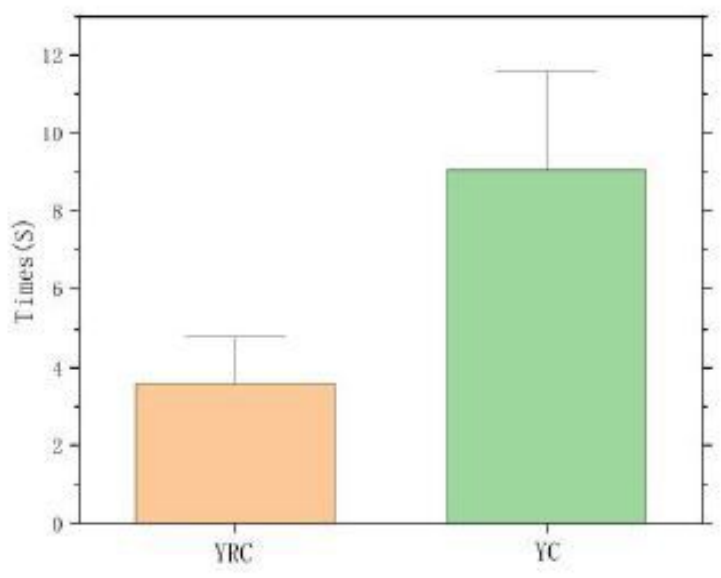

conversion time chart

\section{Figure 4}

The intubation time in the YRC group was shorter than that in the YC group $(89.75 \pm 14.28 \mathrm{~s}$ VS $107.80 \pm 14.96 \mathrm{~s}, \mathrm{p}=0.00$ ) (Figure 4). The ventilation conversion time in the YRC group was shorter than that in the YC group (3.60 \pm 1.20 s VS $9.05 \pm 2.53 s, p=0.00)$ (Figure 4). 